\title{
MODULAR INVARIANCE IN SUPERSYMMETRIC FIELD THEORIES
}

\author{
S FERRARA ${ }^{\text {a b }}$, D LUST ${ }^{a}$, A SHAPERE $^{c}$ and S THEISEN ${ }^{a}$ \\ -CERN,CH-1211 Geneva 23 Switzerland \\ b University of California, Los Angeles, CA 90024, USA \\ - Institute for Adranced Studv, Princeton NJ08540, USA
}

Recerved 8 May 1989

\begin{abstract}
We show how duality invariance of the supergravity action restricts the Kahler potential and the superpotential and connects them to the theory of modular forms This has relevance in string-induced supergravity for those scalar fields which are moduli of the underlying string compactification We also discuss the restrictions imposed on globally supersymmetric theories
\end{abstract}

It is well known $[1,2]$ that the spectrum of a closed string, when compactıfied on a circle of radius $R$, is invariant under the discrete duality transformation $R \rightarrow 1 / 2 R$ In fact, this duality transformation is an exact symmetry to all orders in string perturbation theory, and the moduli space of such compactifications can be taken to be $R \in[1 / \sqrt{2}, \infty)$ instead of $R \in \mathbb{R}^{+}$More generally, the modul space of the heterotic string compactified on a $D$-dimensional torus has locally the structure of the coset space $\mathrm{SO}(16+D$, $D) /[\operatorname{SO}(16+D) \times \mathrm{SO}(D)][3,4]$ However, taking into account the invariance of the spectrum under generalized duality transformations $\mathrm{SO}(16+D, D, \mathbb{Z})$ [5-7] the modulı space is not a coset manifold but actually a fundamental region where points connected by the discrete $\mathrm{SO}(16+D, D, \mathbb{Z})$ transformations are identified This space is in general not a manıfold but has orbifold singularities at points fixed by finite subgroups of $\mathrm{SO}(16+D, D, \mathbb{Z})$ It seems to be a general feature that these special points correspond to compactifications with enhanced gauge symmetry, in addition, the vacuum energy is extremized at isolated fixed points [5] For $D=6$ the resulting effective low energy field theory possesses $N=4$ spacetıme supersymmetry The couplings of the 22 massless $N=4$ vector multıplets (which contain the

\footnotetext{
th This work is supported in part by the United States Department of Energy under contract Nos DE-AA03-76SF00034 (S F ) and DE-AC02-76ERO-2220 (A S )
}

132 moduli of the torus compactification) to supergravity are unıquely described by a non-lınear $\sigma$ model with underlying coset $\mathrm{SO}(22,6) /[\mathrm{SO}(22) \times$ $\mathrm{SO}(6)$ ] [8] Recently, it was also shown $[9,10]$ that duality symmetry is preserved for orbifold compactifications of the heterotic string Aside from this specific context of superstring theories on which we mainly focus in this note, one may investigate the role of duality also in a broader class of field theories and their supersymmetric extensions, as discussed in ref [5]

To study the implications of the modular invar1ance for the supergravity action of massless matter fields, let us consider the simplified model of one chiral multıplet $\phi$ coupled to $N=1$ supergravity We denote the complex scalar component of $\phi$ by $t=2\left(R^{2}+1 b\right)[11,12]$, where $b$ and $R$ are real In a string theory context the parameter $t$ could be, for example, the complex modulus describing two-dımensional torus compactifications [5,7] with background metric $G_{l l}=R^{2} \delta_{l j}(l, J=1,2)$ and internal axion $B_{12}=b[11,12]$ More generally, one may thınk of $t$ as being the modulus whose real part describes the overall scale of a compact six-manifold the string is compactified on, and whose imaginary part is the internal axion The duality transformations are now simply $\operatorname{SL}(2, \mathbb{Z})$ transformations of $t$

$t \rightarrow \frac{a t-1 b}{1 c t+d}, \quad a d-b c=1$ 
In terms of the field $\tau=1 t$ these are the usual SL(2, $\mathbb{Z}$ ) transformations The corresponding non-linear $\sigma$ model is based on the coset space $\mathrm{SU}(1,1) /$ $\mathrm{U}(1)=\mathrm{SL}(2, \mathbb{B}) / \mathrm{U}(1)$ which is isomorphic to the complex upper half-plane $\operatorname{Im} \tau \geqslant 0$ Dividing the upper half-plane by the action of the modular group restricts the modular space of the $\tau$-field to the fundamental domain $\left\{|\tau| \geqslant 1, \quad 0 \leqslant \operatorname{Re} \tau \leqslant \frac{1}{2}, \quad \operatorname{Im} \tau>0\right\}$ $\cup\left\{|\tau|>1,-\frac{1}{2}<\operatorname{Re} \tau<0, \operatorname{Im} \tau>0\right\}$

The standard supergravity action [13] of the $t$-field is completely specified by the Kahler potential $K(t$, $\bar{t})=-n \log (t+\bar{t})$, where the integer $n$ is related to the curvature of $\mathrm{SU}(1,1) / \mathrm{U}(1)$ ( $n=3$ for compactıfication on a six-dimensional manifold) This Kahler potential leads to the correct Kahler metric $K_{t i}=$ $\partial_{t} \partial_{t} K(t, \bar{t})$ of the SU( 1,1$) / \mathrm{U}(1)$ non-linear $\sigma$-model with bosonic action

$S=K_{t \bar{t}} \partial_{\mu} t \partial^{\mu} \bar{t}=\frac{n}{(t+\bar{t})^{2}} \partial_{\mu} t \partial^{\mu} \bar{t}$

Here we have assumed that the superpotential of the $t$-field vanishes In string theory this is true at least perturbatively, reflecting the fact that $t$ is a modulus of the underlying compact six-manifold (For $(2,2)$ compactifications the $t$-field superpotential vanıshes even after taking into account non-perturbative $\sigma$ model corrections In $(0,2)$ compactifications the superpotential may receive non-vanıshıng contributions due to world-sheet instantons [12,14]) The action eq (2) is trivially invariant under $\operatorname{SL}(2, \mathbb{Z})$ duality transformations, since it is invariant under $\operatorname{SL}(2, \mathbb{R})$ due to its geometrical interpretation as coset non-lınear $\sigma$-model If we now add a superpotential $W(t)$, the question of $\operatorname{SL}(2, \mathbb{Z})$ invariance becomes non-trivial In a string theory context we might think that the origin of the superpotential is due to nonperturbative string effects which lift the vacuum degeneracy of the background fields It is easy to show that a non-vanıshing superpotential explicitly breaks $\operatorname{SL}(2, \mathbb{R})$ invariance However, we still want to demand invariance under the duality group $\operatorname{SL}(2, \mathbb{Z})$ since we restrict the parameter domain of integration of $t$ to the fundamental region This requirement gives severe restrictions on the form of the superpotential $W(t)$ and establishes a connection to the theory of modular forms [15] In the following we give some examples

For our first example, let us consider how to imple- ment modular invariance in field theories with global supersymmetry Here the Kahler potential $K(\phi, \bar{\phi})$ and the superpotential $W(\phi)$ are unconnected and the non-linear $\sigma$-model action has the form [6]

$S=\int \mathrm{d}^{4} x \mathrm{~d}^{4} \theta K(\phi, \bar{\phi})+\int \mathrm{d}^{4} x \mathrm{~d}^{2} \theta W(\phi)+\mathrm{h} \mathrm{c}$

The chiral superfield $\phi$ transforms under duality transformations like its scalar component $t$ Then the transformation of its fermionic component $\chi$ is $\chi \rightarrow(1 c t+d)^{-2} \chi$ In order for the globally supersymmetric $\sigma$-model to be $\operatorname{SL}(2, \mathbb{Z})$ invariant we have to demand that the Kahler potential be invariant up to a Kahler transformation The superpotential, being holomorphic, must be modular invariant, $1 \mathrm{e}$

$K(t, \bar{t}) \rightarrow K(t, \bar{t})+f(t)+\bar{f}(\bar{t})$,

$W(t) \rightarrow W(t)$

For the superpotential we can take any polynomial of the modular function $\jmath(q)$ which is given by

$J(q)=\frac{3^{6} 5^{3}}{\pi^{12}} \frac{G_{4}^{3}(q)}{\eta(q)^{12}}=\frac{1}{q}+744+196884 q+$

(A definition of the Eisenstein function $G_{4}(q)$ will be given below $\eta(q)$ is the Dedekind eta-function ) $q$ is related to $t$ via $q=\mathrm{e}^{2 \pi 1 \tau}=\mathrm{e}^{-2 \pi t} J(q)$ has a triple zero at $\tau=1$ and a pole at $\tau=1 \infty$

Let us now turn to the more interesting case of local supersymmetry [13] The Kahler potential and the superpotential are now connected and the matter part of the supergravity lagrangian is now described by a single function

$G(t, \bar{t})=K(t, \bar{t})+\log W(t)+\log \bar{W}(\bar{t})$

The component form of the action is

$$
\begin{aligned}
& \mathrm{e}^{-1} \mathscr{L}=\mathrm{e}^{G}\left[3-G_{t}\left(G_{t \bar{l}}\right)^{-1} G_{\bar{\imath}}\right] \\
& \quad+\left\{\mathrm{e}^{G / 2}\left[-G_{t t}-\left(G_{t}\right)^{2}+G_{t}\left(G_{t \bar{t}}\right)^{-1} G_{\bar{t} \bar{t}}\right] \bar{\chi}_{\mathrm{L}} \chi_{\mathrm{L}}\right. \\
& \left.+\mathrm{e}^{G / 2} \bar{\psi}_{\mu \mathrm{R}} \sigma^{\mu \nu} \psi_{\mu \mathrm{R}}-\mathrm{e}^{G / 2} G_{t} \bar{\psi}_{\mathrm{R}} \gamma \chi_{\mathrm{L}}+\mathrm{h} \mathrm{c}\right\} \\
& +\left(\text { terms not involving } \mathrm{e}^{G}\right)
\end{aligned}
$$

Here we have only written down the terms which arise after adding the superpotential ( $\psi_{\mu}$ is the gravitino) The first two terms correspond to the scalar potential and the Yukawa couplings Because of the appearance of $\mathrm{e}^{G}$ in the above lagrangian we have to demand that $G$ is modular invariant It is then easy to 
check that the action eq (7) is invariant under $\operatorname{SL}(2$, $\mathbb{Z})$ transformations

Modular invariance of $G$ can now be implemented in two different ways The first possibility is that $K(t$, $\bar{t})$ is invariant up to a Kahler transformation which now has to be absorbed by the transformation of the superpotential $W(t)$ To be specific, let us choose again $K(t, \bar{t})=-n \log (t+\bar{t})$ Then all terms in the lagrangian which are not proportional to $\mathrm{e}^{G}$, that is all those we have not displayed in eq (7), are automatıcally $\operatorname{SL}(2, \mathbb{R})$ invariant due to the geometric construction of the non-linear $\sigma$-model On the other hand, the terms in eq ( 7$)$ can never be $\operatorname{SL}(2, \mathbb{R})$ invariant However, $\operatorname{SL}(2, \mathbb{Z})$ invariance can be maintained if the superpotential transforms under modular transformations like a modular function of weight $-n$, up to a $t$-independent phase, 1 e if

$W(t) \rightarrow \mathrm{e}^{1 \alpha(a b c d)}(1 c t+d)^{-n} W(t)$

Let us study this situation more carefully $G_{t}$ is given by

$G_{t}(t, \bar{t})=-\frac{n}{t+\bar{t}}+\frac{\partial \log W}{\partial t}$

Since $G$ is modular invariant, $G_{t}$, which is non-holomorphic, must transform with weight 2 , $1 \mathrm{e}$ $G_{T} \rightarrow(1 c t+d)^{2} G_{t} \partial_{t} \log W$ on the other hand is holomorphic but transforms non-covariantly under modular transformations

$\partial_{t} \log W(t)$

$$
\rightarrow(1 c t+d)^{2} \partial_{t} \log W(t)-1 n c(1 c t+d)
$$

Functions with exactly these transformation properthes are known from the theory of modular forms Consider the Eisenstein functions $G_{2 h}(\tau)[15,17]$

$$
G_{2 h}(\tau)=\sum_{m \in \in \mathbb{Z}}^{\prime}(m \tau+n)^{-2 h}
$$

For $k>1$ these are holomorphic functions of modular weight $2 k$ For $k=1$ however, the sum does not converge and a necessary regularization procedure leads to two alternative definitions of $G_{2}$

$$
\begin{aligned}
& \hat{G}_{2}(\tau)=\sum_{m n}^{\prime} \lim _{s \rightarrow 0}(m \tau+n)^{-2}|m \tau+n|^{-3}, \\
& G_{2}(\tau)=2 \zeta(2)+2 \sum_{m=1}^{\infty} \sum_{n=-\infty}^{\infty}(m \tau+n)^{-2}
\end{aligned}
$$

The regularization destroys either holomorphicity or modular covariance $\hat{G}_{2}$ s of weight two but not holomorphic whereas $G_{2}$ is holomorphic and transforms under $\operatorname{SL}(2, \mathbb{Z})$ as

$G_{2}(t) \rightarrow(1 c t+d)^{2} G_{2}(t)-2 \pi 1 c(1 c t+d)$

$G_{2}$ and $\hat{G}_{2}$ are related by

$\hat{G}_{2}(t)=G_{2}(t)-\frac{\pi}{\operatorname{Re} t}$

This is exactly what we need for the construction of the supergravity action Namely, suppose we make the identifications

$\hat{G}_{2}(t)=\frac{2 \pi}{n} G_{t}(t)$

$G_{2}(t)=\frac{2 \pi}{n} \partial_{t} \log W(t)$

Then we obtain the following expression for the superpotential $W(t)$

$$
\begin{aligned}
& W(t)=\exp \left(\frac{n}{2 \pi} \int^{t} \mathrm{~d} t^{\prime} G_{2}\left(t^{\prime}\right)\right)=[\eta(t)]^{-2 n} \\
& =\mathrm{e}^{n \pi t / 6}\left[1+2 n \mathrm{e}^{-2 \pi n t}+2 n(2+n) \mathrm{e}^{-4 \pi n t}+\right],
\end{aligned}
$$

which is of the type one expects from non-perturbative string effects Supersymmetry is unbroken for $\partial_{i}\left(\mathrm{e}^{G / 2}\right)=0$ This occurs at the two orbifold points $t=1$ and $t=\mathrm{e}^{-\pi / 6}$ of the fundamental region for $t$ which are the zeros of $G_{t}$ At these points the superpotential is finite [since the only zero of $\eta(t)$ is at $t=\infty]$ and the cosmological constant is extremized In fact, the scalar potential will always be extremized at these two points [5] The gravitino mass is given by

$\mathrm{e}^{G(t)}=\frac{1}{(t+\bar{t})^{n}}|\eta(t)|^{-4 n}$

Note that this is the one-loop partition function of the bosonic string in $2 n$ transverse dimensions

Let us now turn to the second possibility to obtain a modular invariant $G(t, \bar{t})$ This 1 given by the choice of separately modular invariant expressions for $K(t, \bar{t})$ and $W(t)$ One possibility along these lines is to replace in $K=-\log (t+\bar{t})^{n} \sim-\log V\left(V \sim R^{2 n}\right.$ is the volume of the $2 n$-dimensional internal space) the 
volume $V$ by a modular invariant expression which behaves in the limit $R \rightarrow \infty$ as $R^{2 n}$ and in the limit $R \rightarrow 0$ as $R^{-2 n}$ [6] For the simple case considered here, the ansatz of ref [6] gives

$$
\begin{aligned}
& K(t, \bar{t})=-2 n \\
& \times \log \left[\sum_{p \in \mathbb{Z}} \exp \left(-\frac{\pi}{\operatorname{Re} t}|p+1 q t|^{2}\right)\right]
\end{aligned}
$$

Other interesting examples of this kind of scenario are the no-scale supergravity theories [18] with vanishing scalar potential $\mathrm{e}^{G}\left(-3+G_{i} G_{\bar{t}}^{-1} G_{\bar{t}}\right) \equiv 0$ This leads to broken supersymmetry with vanishing cosmological constant if the superpotential is a constant and the Kahler potential is chosen as $K(t$, $\bar{t})=-3 \log [F(J(t))+\bar{F}(\bar{J}(\bar{l}))]$, where $F$ is such that Re $F>0$ for $t$ in the fundamental domain

In closing, we would like to mention further extensions of this analysis It is clearly trivial to extend it to theories with $N$ chiral superfields $t_{t}$ with Kahler potential $G=\sum_{t=1}^{1} G_{t}\left(t_{t}, \bar{t}_{t}\right)$ Then duality invariance extends to an invariance under $(\operatorname{SL}(2, \mathbb{Z}))^{v}$ This, e $\mathrm{g}$, applies to toroldal compactification of two dimensions with $N=2[5,7]$ It 1s, however, more interesting to consider cases where the moduli space does not have this simple product structure As discussed in refs [6,5], for background fields

$G=\left(\begin{array}{ll}g & 0 \\ 0 & g\end{array}\right), \quad B=\left(\begin{array}{rr}0 & b \\ -b & 0\end{array}\right)$,

where $g$ and $b$ are symmetric $n \times n$ matrices, the relevant duality transformations are generated by elements of the symplectic modular group $\operatorname{Sp}(2 n, \mathbb{Z})$ acting on the complex matrix $b+1 g$ Therefore, the constraint of modular invariance of the supergravity action is related to the theory of modular functions on Riemann surfaces of genus $n$

We can also imagine several other, less straightforward extensions One interesting problem is the inclusion of gauge fields and the dilaton multiplet and the relation of gauge and modular invariance in the corresponding supergravity theories Another is to understand the role played by duality in theories based on Calabı-Yau or $K_{3}$ compactıfications

We thank C Kounnas, H Oogur1, M Porratı, N Seıberg and $G$ Veneziano for discussions

\section{References}

[1] K Kıkkdwa and M Yamasakı, Phys Lett B 149 (1984) 357

[2] V P Natr, A Shapere, A Stromınger and F Wilczek Nucl Phys B 287 ( 1987 ) 402

[3] K Narain, Phys Lett B 169 (1986) 41

[4] K Naraın, M Sarmadı and E Witten, Nucl Phys B 279 (1987) 369

[5] A Shapere and F Wilczek, Nucl Phys B 320 (1989) 669

[6] A Giveon, E Rabinovicı and $G$ Veneziano, preprint CFRN-TH 5106/88

[7] R Dijkgraaf, E Verlınde and H Verlınde, Commun Math Phys 115 (1988) 649

[8] J -P Derendinger and S Ferrara, in Supersymmetry and supergravity '84, eds B de Wit et al (World Scientıfic, Singapore, 1984) p 159

[9] M Dine, $P$ Huet and N Selberg, preprint IASSNS-HEP88/54, CCNY-HEP-88/20

[10] J Lauer, J Mas and H P Nilles, preprint MPI-PAE/PTh 19/89, TUM-TH-102/89 (April 1989)

[11] E Witten, Phys Lett B 155 (1985) 151

[12] M Dine, N Seiberg, $X G$ Wen and E Witten, Nucl Phvs B 278 (1986) 769, B 289 (1987) 357

[13] E Cremmer, B Julıa, J Scherk, S Ferrara, L Girardello and P van Nieuwenhuizen, Nucl Phys B 147 (1979) 105, E Cremmer S Ferrara, I Girardello and A van Proeyen, Nucl Phys B 212 (1983) 413

[14] N Seıberg, in Superstrings '87, eds L Alvarez-Gaume et al (World Scientıfic, Singapore, 1987) p 215

[15] See eg B Rankın, Modular forms and functions (Cambridge U P, Cambridge, 1977),

B Schoeneberg, Ellıptic modular functions (Springer, Berlın, 1970)

[ 16] B Zumino, Phys Lett B 87 (1979) 203

[17] A N Schellekens and N P Warner, Phys Lett B 177 (1986) 317, Nucl Phys B 287 (1987) 317

[18] E Cremmer, S Ferrara, K Kounnas and D V Nanopoulos, Phys Lett B 133 (1983) 61,

J Ellis, A B Lahanas, D V Nanopoulos and K Tamvakıs, Phys Lett B 143 (1984) 429,

J Ellıs, K Kounnas and D V Nanopoulos, Nucl Phys B 247 (1984) 373 